\section{Polymeric Materials}

\section{4-26 September 2008 \\ Halle- Wittenberg, Germany}

Polymeric Materials 2008 (P2008) will take place from 24-26 September 2008 at the Martin Luther University, Halle-Wittenberg. As with previous meetings in this series, this event is a essential feature in the calendars for all those interested in polymer science and engineering.

The technical program will include symposia with main (key) lectures and short contributions as well as poster sessions to the following topics:

- $\quad$ structure and morphology

- polymer characterization and testing

- $\quad$ synthesis and properties

- nanostructured and functional polymers

- polymer applications

- modification and processing

- polymers for biomedical applications

- simulation and modeling and structures and properties

www.physik.uni-halle.de/P2008

\section{Molecular Modeling and Drug Design}

\author{
10-14 September 2008, Istanbul, Turkey
}

\section{A Summer School on Molecular Modeling and Drug}

Design will be held in Istanbul, Turkey, on 10-14 September 2008. This school is organized by Yeditepe University, Faculty of Pharmacy, and supported by the Turkish Association of Pharmaceutical and Medicinal Chemistry.

The organizers invite Ph.D. students, diploma/master students, as well as young scientist at the beginning of their careers in academic or industrial drug research to participate in this summer school. Leading scientists from universities and pharmaceutical companies will present innovative ideas, modern methods and strategies, and best practices, as well as case studies in the field of drug design. The main topics are as follows:

- target identification and drug discovery process

- modern strategies for lead finding and optimization

- computer-assisted methods:

- virtual screening,

- structure-based design,

- 3D QSAR,

- neuronal networks
- in silico ADME-tox and metabolism

- cheminformatics/bioinformatics and synthetic feasibility

- plant protection and biocatalysis research

\section{Confirmed key lecturers}

- Hugo Kubinyi (Germany)

- Thierry Langer (University of Innsbruck, Austria)

- Wolfgang Sippl (University of Halle, Germany)

- Stefano Moro (University of Padova, Italy)

- Gerhard F. Ecker (University of Wien, Austria)

- UÃur Sezerman (Sabanci University, Turkey)

- Klaus-Juergen Schleifer (BASF, Germany)

- Maurizio Botta (University of Siena, Italy)

- Serdar Kuyucak (University of Sydney, Australia)

Additionally, the participants are invited to present their scientific results on posters. The language of the meeting is English.

For more information, contact Mine Yarim <myarim@summerschoolmmdd2008. org.

通 www.summerschoolmmdd2008.org 